\title{
Spin fluid spacetimes from static general relativistic solutions
}

\author{
J P Krisch† and L L Smalley \\ † Department of Physics, University of Michigan, Ann Arbor, M1 48109, USA \\ ‡ Department of Physics, University of Alabama in Huntsville, Huntsville, AL 35899, USA
}

Received 26 August 1992, in final form 4 December 1992

\begin{abstract}
A method for generating spin fluid solutions to the field equations in a RiemannCartan spacetime is presented. The method uses any general relativistic static perfect fluid solution as input. The resulting RC solutions are irrotational and stationary. They will support a non-zero spin density. The spin density has zero Fermi derivative and will not contribute to a circulation.
\end{abstract}

PACS numbers: 0420,0450

\section{Introduction}

Exact solutions to the gravitational field equations are useful, not only for the evaluation of actual physical situations, but also in comparison with each other. The same spacetime metric can result from many matter contents and even from different theories of spacetime structure. Understanding the similarities and differences of theories that can build identical metrics will help our large scale understanding of gravitational processes.

In investigating a metric that can arise from different matter contents or theories, it is often convenient to consider matter with spin. Spinning matter is useful because simple perfect fluids, fluids with viscosity, heat flow or other similar properties [1] and spin fluids can generate the same metric [2]. It is also of interest since spin can activate the torsion that distinguishes two of the currently competing theories of gravitation: the standard Riemannian general relativity (GR) and its extension to spacetimes with torsion, Riemann-Cartan (RC) spacetimes.

Spin has been of interest in relativity for some time. The review articles of Hehl [3] and Hehl et al [4] contain references to many early investigations. Of particular interest is the work of Weyssenhoff and Raabe [5] and Weyssenhoff [6]. A great deal of the work on a relativistic treatment of spin has focused on Lagrangian-based variational calculations. Lagrangian-based theories have been discussed in general by Kopczynski [7], Bailey [8], Israel [9] and Israel and Bailey [10]. These discussions have focused on general relations that such theories must satisfy by Noether's theorem. A specific Lagrangian density is not assumed. Fluids with intrinsic spin also have been discussed by Unal and Vigier [11], Aymard [12], and Gursey [13]. Halbwachs [14] developed a specific Lagrangian describing spinning fluids in special relativity. Ray and Smalley generalized the work of Halbwachs to describe fluids with spin in GR [15] and also in RC spaces with torsion [16-18]. Their work was based on a specific Lagrangian density and produced a variationally derived stress-energy tensor. In their 
first development, spin was not included as a thermodynamic variable. The resulting stress-energy content was that of the classical Weyssenhoff fluid. Later calculations [16-18] included spin as a thermodynamic variable. The development which includes the thermodynamics of spin is referred to as the 'self-consistent' formalism [19]. Several other authors have also discussed the development of a spin fluid stress-energy content which can be used in predictive model calculations. Kopczynski [20] uses a variational method which assumes a stress-energy form. The treatment of Obukhov and Korotky [21], which is Lagrangian-based, differs in significant detail from the work of Ray and Smalley. A detailed comparison of the two methods is given in [18]. Other variationally derived stress-energy contents have been given by Amorin [22] and de Ritas et al [23].

In this paper, using the self-consistent stress-energy content of Ray and Smalley, we present a method of generating solutions to the axis-symmetric field equations in an RC spacetime. The method uses any static general relativistic solution as input. The output solutions describe matter with general relativistic fluid vorticity and an intrinsic spin content. The RC vorticity is zero. The field equations associated with the method can of course also be solved for new Einstein-Cartan solutions which would then also be solutions in static general relativity. The method is interesting, not only because of the intrinsic interest of interpreting exact solutions to the field equations, but also because it generates some interesting insights into the idea of static versus stationary metrics in RC spaces.

In the next section we briefly review the spin fluid formalism and the structure of the field equations, describe the generating method and discuss RC spaces with spin density. An example is given in the last section.

\section{Formalism}

\subsection{Spin fluids}

A spin fluid is a fluid with an angular momentum density $S_{a b}$ defined throughout its extent. The stress-energy tensor derived by Ray and Smalley [16-18] from the Lagrangian variation can be written as the sum of two parts:

$$
T_{a b}=T_{a b} \text { (spin) }+T_{a b} \text { (fiuid) }
$$

where $T_{a b}$ (fluid) is the perfect fluid stress-energy tensor

$$
T_{a b}(\text { fluid })=(\varepsilon+p) U_{a} U_{b}+p g_{a b}
$$

and $\varepsilon$ is the energy density and $p$ is the pressure.

The spin fluid portion of the stress-energy tensor is

$$
T_{a b}(\text { spin })=2 U_{(a} S_{b) c} \dot{U}^{c}+\nabla_{c}^{*}\left[U_{(a} S_{b)}{ }^{c}-\tilde{\omega}_{c(a} S_{b)}{ }^{c}\right] \text {. }
$$

$\tilde{\omega}_{a b}$ is the angular velocity associated with the spin. It is defined in terms of an orthonormal tetrad $A_{a}^{(\mu)}$ chosen so that $A_{a}^{(0)}$ lies along the fluid velocity.

$$
\tilde{\omega}_{a b}=A_{[a}^{(\mu)} A_{b](\mu)} \text {. }
$$

The spin density is constrained by the Frenkel [24] condition

$$
U^{a} S_{a b}=0
$$

with $U^{a}$ the fluid velocity. The spin density Fermi-Walker transports along the fluid flow

$$
\frac{\mathrm{D} S_{a b}}{\mathrm{~d} \tau}=-\dot{U}_{c}\left(U_{a} S_{b}^{c}+U_{b} S_{a}^{c}\right)
$$


The spin density $S_{a b}$ is related to the proper torsion $\hat{S}_{a b}{ }^{c}$ by

$$
\hat{S}_{a b}^{c}=\frac{1}{2} S_{a b} U^{c}
$$

This is the Weyssenhoff convective form.

The proper torsion is the trace-free part of the torsion $S_{a b}{ }^{c}$

$$
\hat{S}_{a b}{ }^{c}=S_{a b}{ }^{c}+\frac{2}{3} \delta_{[a}^{c} S_{b] j}^{j} \text {. }
$$

The quantity $S_{b j}{ }^{j}$ is the torsion vector and describes that part of the torsion that does not satisfy the Frenkel condition. It is zero in our calculation. The $\nabla_{b}^{*}$ derivative is $\nabla_{b}^{*}=\nabla_{b}+2 S_{b j}^{j}$. Although the stress-energy tensor was constructed for self-consistent fluids in a Riemann-Cartan spacetime, it is also valid in a self-consistent general relativity. The only difference is in the derivative operator $\nabla_{b}^{*}$. In general relativity it is the ordinary covariant derivative. For zero torsion vector, it is also the covariant derivative in the Einstein-Cartan theory with the additional spin connection calculated from the torsion.

$$
\Gamma_{a b}^{c}=S_{a b}^{c}-S_{b a}^{c}+S_{a b}^{c} .
$$

The field equations in a Riemann-Cartan spacetime may be written as

$$
G_{a b}=T_{a b}+\nabla_{c}^{*}\left(S_{a b}^{c}+S_{a b}^{c}-S_{b a}^{c}\right)
$$

\subsection{Field equations}

The metric that we consider is the general axially-symmetric metric

$$
\mathrm{d} s^{2}=-f \mathrm{~d} t^{2}-2 k \mathrm{~d} \phi \mathrm{d} t+\left(D^{2}-k^{2} / f\right) \mathrm{d} \phi^{2}+\mathrm{e}^{\mu} \mathrm{d} r^{2}+\mathrm{e}^{\beta} \mathrm{d} z^{2} .
$$

The tetrads that diagonalize this metric are

$$
\begin{array}{ll}
A_{a}^{(0)}=\left(\sqrt{f}, 0,0, \frac{k}{\sqrt{f}}\right) & A_{a}^{(1)}=\left(0, e^{\mu / 2}, 0,0\right) \\
A_{a}^{(2)}=\left(0,0, e^{\beta / 2}, 0\right) & A_{a}^{(3)}=\left(0,0,0, \frac{D}{\sqrt{f}}\right) .
\end{array}
$$

The tetrad indexed Einstein tensors for this metric in a Riemann-Cartan spacetime are

$$
\begin{aligned}
G_{(11)}= & \mathrm{e}^{-\mu}\left(\frac{D_{r} f_{r}}{2 D f}-\frac{f_{r}^{2}}{4 f^{2}}+\frac{D_{r} \beta_{r}}{2 D}+\frac{\Omega_{r}^{2}}{4 D^{2}}\right) \\
& +\mathrm{e}^{-\beta}\left[-\frac{D_{z} f_{z}}{2 D f}+\frac{f_{z}^{2}}{4 f^{2}}-\frac{D_{z} \beta_{z}}{2 D}+\frac{D_{z z}}{D}-\Omega_{z}\left(\frac{f k_{z}-k f_{z}}{f}+\sqrt{f} S_{z \phi}\right) / 4 D^{2}\right] \\
G_{(22)}= & \mathrm{e}^{-\beta}\left(\frac{D_{z} f_{z}}{2 D f}-\frac{f_{z}^{2}}{4 f^{2}}+\frac{D_{z} \mu_{z}}{2 D}+\frac{\Omega_{z}^{2}}{4 D^{2}}\right) \\
& +\mathrm{e}^{-\mu}\left[-\frac{D_{r} f_{r}}{2 D f}+\frac{f_{r}^{2}}{4 f^{2}}-\frac{D_{r} \mu_{r}}{2 D}+\frac{D_{r r}}{D}-\Omega_{r}\left(\frac{f k_{r}-k f_{r}}{f}+\sqrt{f} S_{r \phi}\right) / 4 D^{2}\right] \\
G_{(33)}= & \mathrm{e}^{-\mu}\left[\frac{D}{2 f}\left(\frac{f_{r}}{D}\right)+\left(\frac{\beta_{r}-\mu_{r}}{4}\right)\left(\frac{f_{r}}{f}+\beta_{r}\right)+\frac{\beta_{r r}}{2}+\frac{D_{r} f_{r}}{2 D f}-\frac{f_{r}^{2}}{4 f^{2}}+\frac{\Omega_{r}^{2}}{4 D^{2}}\right] \\
& +\mathrm{e}^{-\beta}\left[\frac{D}{2 f}\left(\frac{f_{z}}{D}\right)+\left(\frac{\mu_{z}-\beta_{z}}{4}\right)\left(\frac{f_{z}}{f}+\mu_{z}\right)+\frac{\mu_{z z}}{2}+\frac{D_{z} f_{z}}{2 D f}-\frac{f_{z}^{2}}{4 f^{2}}+\frac{\Omega_{z}^{2}}{\left.4 D^{2}\right]}\right.
\end{aligned}
$$




$$
\begin{aligned}
G_{(00)}=\mathrm{e}^{-\mu}\left[\frac{D}{2 f}\right. & \left(\frac{f_{r}}{D}\right)_{r}+\left(\beta_{\mathrm{r}}-\mu_{r}\right)\left(\frac{f_{r}}{4 f}-\frac{D_{r}}{2 D}\right)+\frac{\mu_{r} \beta_{\mathrm{r}}}{4}-\frac{\beta_{r r}}{2}-\frac{\left(\beta_{r}\right)^{2}}{4} \\
& \left.-\frac{D_{r r}}{D}+\frac{3 D_{r} f_{r}}{2 D f}-\frac{3 f_{r}^{2}}{4 f^{2}}+\Omega_{r}\left(3 \frac{f k_{r}-k f_{r}}{f}-\sqrt{f} S_{\mathrm{r \phi}}\right) / 4 D^{2}\right] \\
& +\mathrm{e}^{-\beta}\left[\frac{D}{2 f}\left(\frac{f_{z}}{D}\right)_{z}+\left(\mu_{z}-\beta_{z}\right)\left(\frac{f_{z}}{4 f}-\frac{D_{z}}{2 D}\right)+\frac{\mu_{z} \beta_{z}}{4}-\frac{\mu_{z z}}{2}-\frac{\left(\mu_{z}\right)^{2}}{4}\right. \\
& \left.-\frac{D_{z z}}{D}+\frac{3 D_{z} f_{z}}{2 D f}-\frac{3 f_{z}^{2}}{4 f^{2}}+\Omega_{z}\left(3 \frac{f k_{z}-k f_{z}}{f}-\sqrt{f} S_{z \phi}\right) / 4 D^{2}\right]
\end{aligned}
$$

$$
G_{(03)}+G_{(30)}=\mathrm{e}^{-(\mu+\beta) / 2}\left\{\frac{\left[\left(\left(f k_{r}-k f_{r}\right) / D\right) \mathrm{e}^{(\beta-\mu) / 2}\right]_{r}}{f}-\frac{\left[\left(f S_{r \phi} / D\right) \mathrm{e}^{(\beta-\mu) / 2}\right]_{r}}{\sqrt{f}}\right.
$$$$
\left.+\frac{\left[\left(\left(f k_{z}-k f_{z}\right) / D\right) \mathrm{e}^{(\mu-\beta) / 2}\right]_{z}}{f}-\frac{\left[\left(f S_{z \phi} / D\right) \mathrm{e}^{(\mu-\beta) / 2}\right]_{z}}{\sqrt{f}}\right\}
$$

$R_{r z}+R_{z r}=\frac{\mu_{z} D_{r}}{D}+\frac{\beta_{r} D_{z}}{D}-2 \frac{D_{r z}}{D}+\frac{D_{z} f_{r}}{D f}+\frac{D_{r} f_{z}}{D f}-\frac{f_{r} f_{z}}{f^{2}}+\frac{f k_{r}-k f_{r}}{2 f D^{2}} \Omega_{z}+\frac{f k_{z}-k f_{z}}{2 f D^{2}} \Omega_{r}$

where

$$
\Omega_{r}=\frac{f k_{r}-k f_{r}}{f}-\sqrt{f} S_{r \phi} \quad \text { and } \quad \Omega_{z}=\frac{f k_{z}-k f_{z}}{f}-\sqrt{f} S_{z \phi} .
$$

The field equations for a perfect spin fluid matter content are

$$
\begin{aligned}
& G_{(00)}=\varepsilon \\
& G_{(11)}=p+\mathrm{e}^{-\mu} \frac{\sqrt{f} S_{r \phi}}{2 D^{2}} \Omega_{r} \\
& G_{(22)}=p+\mathrm{e}^{-\beta} \frac{\sqrt{f} S_{z \phi}}{2 D^{2}} \Omega_{z} \\
& G_{(33)}=p+\mathrm{e}^{-\mu} \frac{\sqrt{f} S_{r \phi}}{2 D^{2}} \Omega_{r}+\mathrm{e}^{-\beta} \frac{\sqrt{f} S_{z \phi}}{2 D^{2}} \Omega_{z} \\
& R_{r z}+R_{z r}=\frac{\sqrt{f} S_{r \phi}}{2 D^{2}} \Omega_{z}+\frac{\sqrt{f} S_{z \phi}}{2 D^{2}} \Omega_{r} \\
& G_{(03)}+G_{(30)}=\frac{3 S_{r \phi} f_{r} \mathrm{e}^{-\mu}}{2 D \sqrt{f}}+\frac{3 S_{z \phi} f_{z} \mathrm{e}^{-\beta}}{2 D \sqrt{f}}
\end{aligned}
$$

with $\varepsilon$, the energy density, $p$ the pressure and $S_{i j}$ the spin density of the fluid. We consider only spin components in the radial and $z$-directions. We assume the spin density $S_{r z}$ is zero.

\subsection{Generating method and spin}

The special solution that we will study imposes the conditions $\Omega_{r}=0, \Omega_{z}=0$

$$
S_{\mathrm{r \phi}}=\frac{f k_{r}-k f_{r}}{\sqrt{(f)^{3}}} \quad \text { and } \quad S_{\mathrm{z \phi}}=\frac{f k_{z}-k f_{z}}{\sqrt{(f)^{3}}} .
$$

These conditions are equivalent to requiring the fluid vorticity in the RC space to be zero. 
If we had included $S_{r z}$ as a possible non-zero spin density, the lack of $\phi$-dependence in the metric and the definitions (25) would have enforced a zero. With these conditions, the diagonal Einstein tensors, the $(r z)$ Einstein tensors and the associated field equations (19)-(23) are formally identical to those found in static general relativity for the metric

$$
\mathrm{d} s^{2}=-f \mathrm{~d} t^{2}+\mathrm{e}^{-\mu} \mathrm{d} r^{2}+\frac{D^{2}}{f} \mathrm{~d} \phi^{2}+\mathrm{e}^{-\beta} \mathrm{d} z^{2}
$$

and for a perfect fluid with pressure $p$ and energy density $\varepsilon$.

The remaining (03) field equation, (24) becomes

$$
\mathrm{e}^{-\mu}\left(f k_{r}-k f_{r}\right) f_{r}+\mathrm{e}^{-\beta}\left(f k_{z}-k f_{z}\right) f_{z}=0 .
$$

This equation is especially interesting. Rewriting it in terms of the spin and accelerations, one finds that it takes the form

$$
\dot{U}_{a} S_{b}^{a}=0 \text {. }
$$

The spin is orthogonal to the fluid acceleration. From equation (6), this implies that the Fermi derivative of the spin is zero. Equation (27) also implies that the spin contribution to the fluid current with a potential representation is zero $[17,23,25]$. Since it is this current which could enter into a calculation of the circulation [26], the spin will not contribute to the circulation for these solutions.

It is also interesting that (25) can be rewritten as

$$
\beta_{a}=\frac{S_{\alpha \phi}}{\sqrt{f}}=\left(\frac{k}{f}\right)_{, a} .
$$

The vector $\beta_{a}$ is hypersurface orthogonal to the surfaces of fuid irrotationality in a GR spacetime. The vector $\beta_{a}$ was discussed by Landau and Lifshitz [27] in a discussion of Coriolis effects on particle motion in constant gravitational fields. Greene et al [28] have pointed out that $\beta_{a}$ is related to the rate of rotation of a Killing based frame relative to one that is Fermi-Walker transported.

In the next section, we will discuss whether the solution is static or stationary.

\subsection{The static condition and spin}

In general relativity, a static solution is one which has a hypersurface orthogonal timelike Killing vector $\xi^{a}$ [29]

$$
\begin{aligned}
& \xi^{a} \xi_{a}=g_{00}<0 \\
& \xi_{(a ; b)}=0 \\
& \xi_{[a ; b} \xi_{c]}=0 .
\end{aligned}
$$

The last condition implies that $\xi_{a}$ is proportional to the gradient of a scalar function, $\sigma[30]$

$$
\xi_{a}=\operatorname{constant}(\sigma)_{, a} .
$$

In general relativity, a vector that is locally hypersurface orthogonal is also irrotational. Since in general relativity, the two statements imply each other, irrotationality is often given as the condition for a spacetime to be static. Greene et al [28] have discussed the conditions for a spacetime to be synchronizable. Ehlers and Kundt [31] have expressed the conditions for a spacetime to be static in terms of the fluid velocity. 
In spacetimes with torsion, the condition for the Killing vector to be the gradient of a scalar is complicated by the antisymmetric connection. Since torsion is usually activated by rotation and the presence of spin, inquiring into the condition for a spacetime with torsion to be static seems to have a trivial answer. However, we have seen in the previous section that in a Riemann-Cartan spacetime, it is possible for the contributions from spin and fluid rotation to cancel each other in all of the diagonal and some of the off diagonal field equations. The fluid vorticity in the RC space is zero; the fluid is irrotational. One may ask the question: is the irrotational RC space generated from the static GR space itself static? Is there a hypersurface orthogonal Killing vector?

From equation $(30 c)$ we can write

$$
\xi_{a}\left(\xi_{c}\right)_{; b}+\xi_{b}\left(\xi_{a}\right)_{i c}+\xi_{c}\left(\xi_{b}\right)_{; a}=0
$$

where

$$
\xi_{a}=(-f, 0,0,-k)
$$

is the usual stationary Killing vector, $\xi_{a}=g_{0 a}$. The twist of this vector for the generated $\mathrm{RC}$ space is zero.

Multiplying through by $\xi_{c}$ and antisymmetrizing we find in an RC spacetime

$$
\left(\frac{\xi_{a}}{\xi^{n} \xi_{n}}\right)_{, b}-\left(\frac{\xi_{b}}{\xi^{n} \xi_{n}}\right)_{, a}=\frac{\xi_{m}}{\xi^{n} \xi_{n}} S_{b a} U^{m} .
$$

$\xi_{a}$ is not expressible as the gradient of a scalar in general. However, if $S_{a b}$ can be written as

$$
\frac{\xi_{m} U^{m} S_{a b}}{\xi^{n} \xi_{n}}=\left(\frac{\psi_{b}}{\xi^{n} \xi_{n}}\right)_{, a}-\left(\frac{\psi_{a}}{\xi^{n} \xi_{n}}\right)_{, b}
$$

then equation (34) becomes

$$
\left(\frac{\xi_{a}-\psi_{a}}{\xi^{n} \xi_{n}}\right)_{, b}=\left(\frac{\xi_{b}-\psi_{b}}{\xi^{n} \xi_{n}}\right)_{, a}
$$

and we have that

$$
\xi_{a}-\psi_{a}=\xi^{n} \xi_{n}(\sigma)_{a} .
$$

$\xi_{a}-\psi_{a}$ is locally hypersurface orthogonal. Using equation (25) one finds that

$$
\psi_{a}=(0,0,0,-k)
$$

Note that because of the Frenkel condition, $U^{a} S_{a b}=0, \psi_{0}$ is zero. The RC vector that is hypersurface orthogonal is identical to the Killing vector of the static GR space.

We have exhibited a hypersurface orthogonal, timelike vector. When the vector, $\xi_{a}-\psi_{a}$, is substituted into Killing's equation, one finds that the condition for this vector to be a Killing vector is that $(k / f)=$ constant. This relation between $k$ and $f$ will zero the spin. The hypersurface orthogonal vector is not a Killing vector in the $\mathrm{RC}$ spacetime, except in the trivial case. The metric is most generally stationary.

\subsection{The generating process}

The statement describing the generating process is: to every static perfect fluid metric in general relativity $\left(g_{a b}, D, p, \varepsilon\right)$, a stationary Riemann-Cartan solution $\left(g_{a b}^{*}, D, p\right.$, 
$\left.\varepsilon, S_{a b}\right)$ can be assigned with

$$
\begin{array}{ll}
g_{a b}^{*}=g_{a b} & a, b=t, r, z \\
g_{0 \phi}^{*}=-k & e^{-\mu}\left(f k_{r}-k f_{r}\right) f_{r}+e^{-\beta}\left(f k_{z}-k f_{z}\right) f_{z}=0 \\
g_{\phi \phi}^{*}=\frac{D^{2}-k^{2}}{f} & \\
S_{a \phi}=\frac{f k_{a}-k f_{a}}{\sqrt{f^{3}}} & a=r, z .
\end{array}
$$

\section{Application}

Barnes [32] has considered solutions of the field equations in general relativity with a static, perfect fluid matter content. Using the method described in section 2 , the metric solutions given by him may be easily generalized to Riemann-Cartan solutions with a spin fluid content. For example, one of his solutions for constant density is a type $\mathrm{D}$ solution with metric

$$
\begin{aligned}
& \mathrm{d} s^{2}=-r^{2} z^{2} \mathrm{~d} t^{2}+r^{2} \mathrm{~d} z^{2}+F(r) \mathrm{d} \phi^{2}+F^{-1}(r) \mathrm{d} r^{2} \\
& F(r)=\frac{A r^{2}}{3} \quad A>0 \quad \text { and } \quad A=-\varepsilon \quad p+\varepsilon=0 .
\end{aligned}
$$

Applying equation (38b) and assuming that $k(r, z)=k(r) k(z)$ we find

$$
\begin{aligned}
& k(r, z)=B r^{2} z^{2} e^{\Sigma} \\
& \Sigma=-\frac{C}{4}\left(\frac{3}{A r^{2}}+z^{2}\right) .
\end{aligned}
$$

$C$ is a separation constant and $B$ is a constant of integration.

The RC metric is

$$
\mathrm{d} s^{2}=-r^{2} z^{2}\left(\mathrm{~d} t+B \mathrm{e}^{\Sigma} \mathrm{d} \phi\right)^{2}+r^{2} \mathrm{~d} z^{2}+F(r) \mathrm{d} \phi^{2}+F^{-1}(r) \mathrm{d} r^{2} .
$$

The spin densities associated with this solution are

$$
S_{r \phi}=\frac{3 B C z \mathrm{e}^{\Sigma}}{2 r^{2} A} \quad S_{z \phi}=-\frac{B C \mathrm{e}^{\Sigma} r z^{2}}{2} .
$$

In general relativity, solutions with negative energy have generally been considered unphysical. Morris and Thorne [33] have however physically discussed negative energy density in squeezed states. Some recent work [34] on the energy conditions in spacetimes with torsion indicates that this type of solution may possibly be physical and not in violation of the energy conditions.

\section{Discussion}

We have exhibited and applied a method that will generate a spin fluid solution in an RC spacetime from any static GR perfect fluid solution. The generated spin fluid source will be described by the Ray-Smalley stress-energy content [18]. Non-trivial solutions 
require $g_{00}$ to depend on at least two coordinates. The passage from GR to RC maintains a twist free Killing vector. It also keeps the identical vector hypersurface orthogonal in both spaces. However, in RC, the hypersurface orthogonal vector is not a Killing vector, except for zero spin. Another of the interesting properties of the generated RC fluid spacetimes is that they are RC-irrotational and stationary. An observer in a boundary matched vacuum spacetime outside of the fluid could however, see a precession of his gyroscope axes relative to distant stars. The effect would depend on the particular RC solution generated and the way that it matched to the vacuum exterior. In the bounding process, the matching of the first and second fundamental forms at the boundary surface will require that the off-diagonal metric components satisfy, among others, relations like $[35,36]$

$$
[k]_{+}=[k]_{-} \quad \text { and } \quad\left[k_{, a}+S_{\phi a}\right]_{+}=\left[k_{, a}\right]_{-}
$$

where we are using the off diagonal component of the metric of equation (11). Clearly one could arrange a special case where the matching, vacuum off-diagonal metric was zero but in general it is not. The vacuum observer can be in a stationary spacetime with all of its usual properties.

The metric of the stationary RC spacetime is clearly generated from the static GR metric by the coordinate transformation

$$
\mathrm{d} t \geqslant \mathrm{~d} t+\frac{k}{f} \mathrm{~d} \phi \quad \mathrm{d} \phi \geqslant \mathrm{d} \phi .
$$

This is a formal substitution. The antisymmetric connection implicit in the RC space is not generated by this transformation but by the identification of the generated metric as describing a spacetime with torsion. The Fermi constant spin density is also not generated by this transformation but is a stated part of the stress-energy content of the generated stationary solution. The method is of interest not only because it broadens the interpretation of existing solutions but also because of the insights it generates into the meaning of static versus stationary in a space with torsion.

\section{References}

[1] Tupper B O J 1983 Gen. Rel. Grav. 15849

[2] Krisch J 1987 J. Math. Phys. 281544

[3] Hehl F W 1973 Gen. Rel. Grav. 4 333; 19745491

[4] Hehl W, von der Heyde P, Kerlick G D and Nester J M 1976 Rev. Mod. Phys. 48393

[5] Weyssenhoff J and Raabe A 1947 Acta Phys. Pol 97

[6] Weyssenhoff J 1947 Acta Phys. Pol. 926

[7] Kopczynski W 1972 Phys. Lett. 39A 219

[8] Bailey I 1979 Ann. Phys., NY 11976

[9] Israel W 1973 Lett. Nuovo Cimento 7860

[10] Bailey I and Israel W 1975 Commun. Math. Phys. 4265

[11] Unal B C and Vigier J-P 1957 C.R. Acad. Sci. Paris 245 1787, 1891

[12] Aymard A 1956 C.R. Acad. Sci. Paris 243 885, 1100; 19572443133

[13] Gursey F 1957 Nuovo Cimento 5784

[14] Halbwachs F 1968 Prog. Theor. Phys. 24291

[15'] Ray J R and Smalley L L 1982 Phys. Rev. D 262619

[16] Ray J R and Smalley L L 1982 Phy's. Rev. Lett. 49 1059; $198350626 \mathrm{E}$

[17] Ray J R and Smalley L L 1983 Phys. Rev. D 271383

[18] Smalley L L and Ray J R 1990 Class. Quantum Grav. 71445

[19] Smalley L L and Ray J R 1986 Gen. Rel. Grav. 18549 
[20] Kopczynski W 1986 Phys. Rev. D 34352

[21] Obukhov Yu N and Korotky V A 1987 Class. Quantum Grav. 41633

Obukhov Yu N and Piskareva O B 1989 Class. Quantum Grav. 6 L15

[22] Amorin A R 1984 Phys. Lett. 104A 259; 1985 Phys. Rev, D 313099

[23] de Ritas D, Lavorgna M, Platania G and Stornaiolo C 1983 Phys. Lett. 95A 425; 1983 Phys. Rev. D $28713 ; 1985311854$

[24] Frenkel J 1926 Z. Phys. 37243

[25] Schutz F 1970 Phys, Rev. D 22762

[26] Taub A H 1959 Arch. Ration. Mech. Anal. 3312

[27] Landau L D and Lifschitz E M 1980 Classical Theory of Fields 4th edn (Oxford: Pergamon) p 252

[28] Greene R D, Schucking E L and Vishveshwara C V 1979 J. Math. Phys. 16153

[29] Kramer D et al 1980 Exact Solutions of Einstein's Field Equations (Cambridge: Cambridge University Press) p 187

[30] Trautman A 1964 Brandeis Summer Institute on Theoretical Physics, Lectures on General Relativity (Englewood Cliffs, NJ: Prentice-Hall) p 131

[31] Ehlers J and Kundt W 1962 Gravitation: An Introduction to Current Research ed Witten (New York: Wiley)

[32] Barnes A 1972 J. Phys. A: Math. Gen. 5374

[33] Morris M S and Thorne K S 1988 Am. J. Phys. 56395

[34] Smalley L L and Krisch J P 1992 Energy conditions in non-Riemannian spacetime Preprint UAH 7-24-92

[35] Arkuszerski W, Kopczynski W and Ponomariev V N 1975 Commun. Math. Phys. 45183

[36] Bonner W B and Vickers P A I98I Gen. Rel, Grav. 1329 